\title{
Sample Preparation Using Broad Argon Ion Beam Milling for Electron Backscatter Diffraction (EBSD) Analysis
}

\author{
Pawel Nowakowski ${ }^{1}$, James Schlenker ${ }^{1}$, Mary Ray ${ }^{1}$ and Paul Fischione ${ }^{1}$ \\ 1. E. A. Fischione Instruments, Inc, 9003 Corporate Circle, Export, PA USA
}

One of the most challenging steps in materials research using electron backscatter diffraction (EBSD) technique is accurate sample preparation. This is because the EBSD signal comes from the top few nanometers (about 20 to $50 \mathrm{~nm}$ ) of the sample [1], which requires a very high quality surface preparation. The sample surface has to be free from contamination, oxidation, and, above all, crystal lattice damage (elastic strain) and plastic deformation (plastic strain) [2].

Several sample preparation techniques can be used to get high quality EBSD patterns, including mechanical polishing and electropolishing. While these techniques are accurate and yield good results, the techniques require several preparation steps and can be quite time consuming (more than ten hours, in some cases) [3]. Another limitation is that plastic strain can be introduced during mechanical grinding/polishing, which leads to degradation of EBSD pattern quality [4]. Other sample preparation methods include focused ion beam (FIB) and broad beam ion milling [5]. Application of high energy $(30 \mathrm{kV}) \mathrm{Ga}$ ions can damage a sample's crystalline structure by introducing lattice defects (strain induction), the implantation of gallium ions, and surface amorphization that completely obstruct the EBSD signal. The development of FIB columns that employ a lower voltage beam (as low as $2 \mathrm{kV}$ ) can improve the quality of EBSD patterns [6,7]. However, this FIB technique is best suited to small sample preparation areas (approximately $50 \times 50 \mu \mathrm{m}$ ) and sample preparation for transmission electron microscopy [7]. Mechanical polishing and FIB techniques can also cause dynamic phase transformation in austenitic steels or steels that contain retained austenite [8].

This work illustrates the advantage of low energy argon ion milling sample preparation for EBSD analyses when compared to mechanical polishing. Two cases are considered:

1) Lattice defect induction (strain induction) by mechanical polishing. A silicon $\langle 001\rangle$ single crystal sample was mechanically polished and then argon ion milled. The kernel average misorientation (KAM) was calculated from EBSD data.

2) Meta-stable austenite phase transformation induced during mechanical polishing of austenitic stainless steel 300 series samples.

All samples were mechanically polished and ion milled using Fischione Instruments' Model 1060 SEM Mill. The samples were then observed with a field emission microscope and analyzed using EBSD technique at $20 \mathrm{kV}$.

Figure 1 shows the KAM distributions for a Si sample mechanically polished and ion milled; at $4 \mathrm{kV}$ and $2 \mathrm{kV}$, broad-beam argon ion milling achieved a mean KAM of $0.04^{\circ}$, which is close to the reference Si EBSD calibration standard. Sample preparations at $4 \mathrm{kV}$ and $2 \mathrm{kV}$ were also characterized by a very narrow KAM distribution when compared to the 2 hours colloidal silica mechanical polishing finish. 
References:

[1] R.A. Schwarzer, D.P. Field, B.L. Adams, M. Kumar, A.J Schwartz Book, Springer, (2009) p .1-20.

[2] S.I. Wright, M.M. Nowell, D.P. Field, Microscopy and Microanalysis, 17, (2011) p. 316-329.

[3] J-P Thomas, C. Chauvy, P. Heurtier, Book, EDP Sciences, (2004) p. 111-118.

[4] D. Katrakova, F. Mucklich, Paer I: Metals, Parc Metallog, 38, (2001) p. 547-565.

[5] L. Ma, Micron, 35 (2004), p. 273-279.

[6] J.R. Michael, Lucille A. Giannuzzi, Microsc Microanal, 13 (2007).

[7] J. Mayer et al, MRS Bulletin, 32 (2007), p. 400-4007.

[8] K.E. Knipling et al, Materials Characterization, 61(2010) p. 1-6.
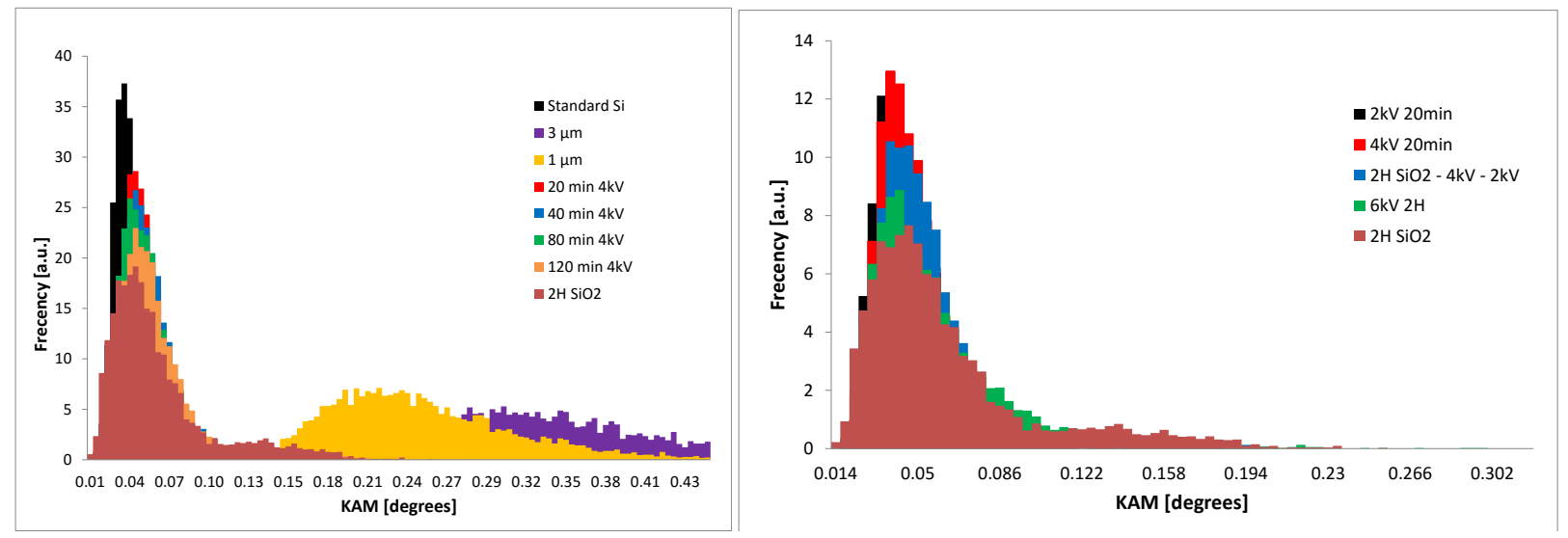

Figure 1. Kernel average misorientation (KAM) distributions (cluster 3 x 3 pixels, $5^{\circ}$ threshold angle) for a Si sample mechanically polished and ion milled at different voltages.

Figure 2 shows the austenitic stainless steel 300 series after mechanical polishing finished by colloidal silica after 3 hours polishing (Figure 2a) and after $4 \mathrm{kV}$ and $2 \mathrm{kV}$ Ar ion milling (Figure 2b). After mechanical polishing, the fraction of austenite in the sample is close to 50\%; after ion milling, the fraction increases to almost $100 \%$.
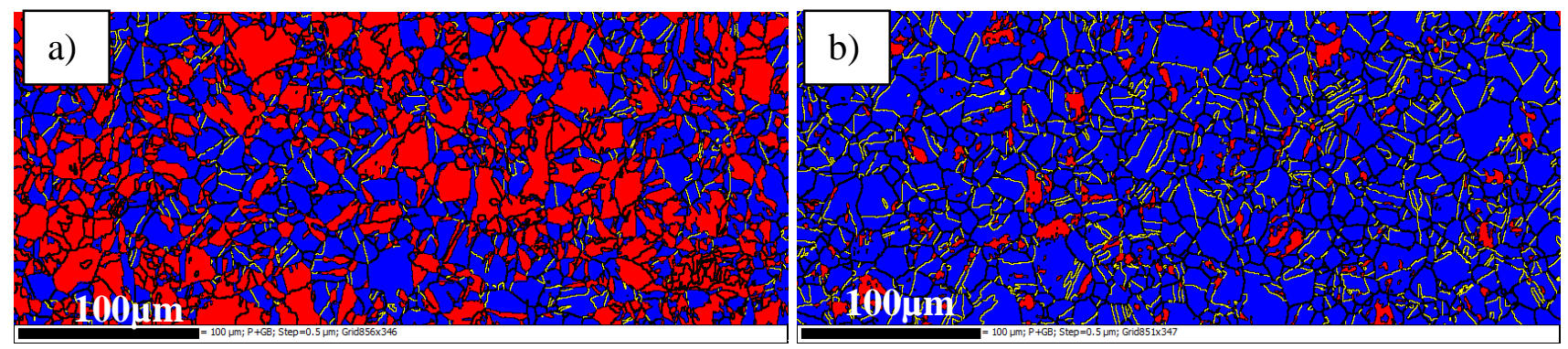

Figure 2. EBSD phase distribution maps (blue - iron FCC, red - iron BCC, black lines - grain boundaries, and yellow lines - tween boundaries within austenite grains): a) 3 hours, colloidal silica mechanical polishing finish, 50\% FCC - 50\% BCC, b) 2 hour $4 \mathrm{kV}$ and 1 hours $2 \mathrm{kV}$ Ar ion milling finish, $95 \% \mathrm{FCC}-5 \% \mathrm{BCC}$. 